\title{
Percepción del aprendizaje según estudiantes de octavo semestre de la licenciatura en Derecho de la Universidad de Sonora Campus Caborca Estudio de caso
}

\author{
García Moraga, Reyna Elizabeth¹, León Félix René Alejandroํ, Torres García Elizabeth² \\ ' Departamento de Ciencias Sociales de la Universidad de Sonora, Campus Caborca \\ 2 Professor and Systems Administrator of Staircase, New Zealand, NZ.
}

\section{Resumen}

Un sistema de educación superior de alta calidad es imprescindible para garantizar que los egresados contribuyan en forma efectiva al desarrollo productivo, económico y social de su entorno. En gran medida las investigaciones en torno a la calidad educativa son realizados por las autoridades de la materia: se aborda la satisfacción académica, los conocimientos, las habilidades, la infraestructura, a partir del ideal que se ha planeado y/o establecido desde la cúspide de la administración y pocas veces se analiza desde la perspectiva de los propios estudiantes. Al margen quedan las expectativas de estos, si se satisfizo o no su necesidad de aprendizaje, si los conocimientos adquiridos corresponden a las exigencias laborales, si sus aptitudes y actitudes son efectivas herramientas para el éxito o pesadas cargas obsoletas. La investigación, a diferencia de otros estudios, se realiza con estudiantes que conservan su calidad como tales y considera a aquellos que cursan el octavo semestre de la licenciatura en Derecho de la Universidad de Sonora Campus Caborca quienes poseen dos características indispensables a). Han cursado la mayor parte del plan de estudios y b). Realizan la segunda de tres prácticas profesionales, en cuyo espacio han llevado a la práctica los haberes académicos obtenidos en aula.

Palabras Clave: Percepción de la calidad, calidad educativa, satisfacción del estudiante.

\section{Perception of learning according to eighth semester students of the Law degree of the University of Sonora Campus Caborca Case study}

\begin{abstract}
A high-quality higher education system is essential to ensure that graduates contribute effectively to the productive, economic and social development of their environment. To a large extent, research on educational quality is carried out by the relevant authorities: academic satisfaction, knowledge, skills and infrastructure are approached, based on the ideal that has been planned and / or established from the top of the administration and rarely analyzed from the perspective of the students themselves. Regardless, their expectations remain, whether their need for learning was met or not, if the knowledge acquired corresponds to labor requirements, if their skills and attitudes are effective tools for success or heavy obsolete burdens. The research, unlike other studies, is carried out with students who retain their quality as such and considers those who are in the eighth semester of the law degree of the University of Sonora Campus Caborca with two essential characteristics a). They have completed most of the curriculum and b). They carry out the second of three professional practices, in whose space they have implemented the academic assets obtained in the classroom.
\end{abstract}

Keywords: Perception of quality, educational quality, student satisfaction. 


\section{INTRODUCCIÓN}

Uno de los aspectos que las Instituciones de Educación Superior en México (IES) analizan y fomentan hoy por hoy, es la calidad académica de los programas de estudio. De hecho, nuestro país cuenta con una larga historia en materia de educación superior, así lo demuestra la creación en 1551 de la Real y Pontificia Universidad de México, que a la postre se convirtió en la Universidad Nacional Autónoma de México (UNAM) (Cruz y Cruz, 2008).

El gobierno federal en México y en general en América Latina, formularon en los años ochenta políticas para evaluar la calidad del desempeño de las universidades, las que experimentaron por vez primera la creciente presión crítica respecto a sus actividades, estructuras y prácticas institucionales, lo que orilló a buscar transformaciones significativas internas (Acosta, 2010)

En el ámbito de la calidad propiamente, la evaluación de la educación superior se institucionalizó con la puesta en marcha del Programa para la modernización educativa federal en el año de 1989 cuyo fin era impulsar la mejora de la calidad en los programas educativos (SEGOB, 2020) Este programa fue resultado de la consulta Nacional para la Modernización de la Educación que se llevó a cabo en el territorio mexicano por instrucciones del entonces presidente de la República, Carlos Salinas de Gortari.

En aquel momento se estableció la necesidad de reforzar la eficiencia y la calidad de la educación superior mediante formas eficientes de trabajo que incrementen la productividad.

Por otra parte, en el año 2002 se creó en México el Instituto Nacional para la Evaluación de la Educación (INEE) como consecuencia del compromiso social-gubernamental por la calidad de la educación. Una parte sustantiva era el establecimiento de los cambios y compromisos propuestos como son el desarrollo de comunidades de aprendizaje exitosas, el cumplimiento de los maestros con su trabajo, el aprovechamietno óptimo de las jornadas escolares, el aprendizaje de un segundo idioma y de computación, todo, considerando que la interrelación académica entre los niveles educativos es una cuestion de calidad a veces poco abordada pero de gran relevancia académicamente (Yzaguirre, 2005).

Sin embargo, con la entrada en vigor de la reforma constitucional en materia educativa publicada en el Diario Oficial de la Federación el 15 de mayo de 2019, que deroga diversas disposiciones de los artículos 3o, 31 y 73 de la Constitución Política de los Estados Unidos Mexicanos, desapareció también este organismo para dar paso a un organismo sustituto denominado Centro Nacional para la Revalorización del Magisterio y la Mejora Continua de la Educación. Precisamente, uno de los grandes problemas que enfrenta la calidad educativa en todos los niveles, es el cambio sexenal de gobierno que modifica o reforma la política educativa en México, sin que ello permita la instauración de una planeación a largo plazo en la materia.

En gran medida las investigaciones en torno a este tema son realizados por las propias autoridades educativas: se aborda la satisfacción académica, los conocimientos, las habilidades, la infraestructura, a partir del ideal que se ha formado y/o establecido desde la cúspide de la administración y pocas veces se analiza desde la perspectiva de los propios estudiantes. ¿Conocemos cuales son sus expectativas? ¿Se satisface o se satisfizo su necesidad de aprendizaje? ¿Los conocimientos adquiridos corresponden a la realidad laboral? ¿Son sus aptitudes y actitudes herramientas para el éxito o pesadas cargas obsoletas? Estas son las preguntas que quedan en el aire desde la perspectiva de los estudiantes, como arriba se señala.

\section{MATERIALES Y MÉTODOS}

El presente estudio es transversal y descriptivo en el que se incluyó a la totalidad de los estudiantes matriculados en el octavo semestre de la Licenciatura en Derecho de la Universidad de Sonora durante el periodo enero-junio de 2017. Para tal efecto se solicitaron las listas de inscritos a dicho semestre a la Coordinación de Servicios Escolares del Campus Caborca. Para llevar a cabo el operativo de levantamiento de encuestas se conformó un equipo de trabajo previamente capacitado y coordinado por los autores. Para captar la información de la encuesta se utiliza un cuestionario que se construyó a partir de la búsqueda y consulta de estudios y herramientas relacionadas con la opinion de los estudiantes sobre diversos aspectos en torno a su permanencia en espacio universitario.

La recolección de datos se realizó por medio de la encuesta mediante el uso de cuestionarios aplicados por personal capacitado.

La investigación, a diferencia de otros estudios, se realiza en tanto aún conservan su calidad de estudiantes y comprende a los estudiantes de octavo semestre de la licenciatura en Derecho quienes poseen dos características indispensables para el presente: A) Han cursado la mayor parte del plan de estudios y B) Se encuentran realizando la segunda de tres prácticas profesionales, en cuyo espacio han llevado a la práctica los haberes académicos obtenidos en aula. 


\section{RESULTADOS Y DISCUSIÓN}

El plan de estudios de la Licenciatura en Derecho de la Universidad de Sonora data del año 2004. Según los antecedentes institucionales, se tomó el diseño basado en competencias como la metodología apropiada para la reestructuración del indicado plan, atendiendo a la congruencia derivada del Modelo Educativo de la Universidad de Sonora cuyos lineamientos enfatizan la importancia de que los nuevos planes de estudio reúnan las siguientes características:

1. Procuren una formación básica sólida

2. Posean flexibilidad en las rutas curriculares

3. Estén centrados en el autoaprendizaje del alumno

4. Estén comprometidos con la realidad social del país.

5. Se vinculen de manera clara con los sectores productivo y social.

6. Que sean comprometidos con el estudiante

7. Incluyan modalidades no convencionales de enseñanza

8. Sean estructurados alrededor de cinco ejes formativos generales (común, básico, profesional, especializante e integrador).

Para la elaboración del Plan se definió primeramente al Derecho como la ciencia que estudia al conjunto de normas jurídicas, general y específicas- que regulan las conductas externas entre los individuos, grupos e instituciones, con el fin de obtener su convivencia armónica, a partir del uso de recursos teóricos, metodológicos y estratégicos.

Los planes y programas de estudio constituyen en si mismos el ideal a alcanzar para formar profesionales del Derecho. Sin embargo, no existen a la fecha estudios dentro de la Universidad de Sonora que contraste la realidad con la teoría o con lo efectivamente alcanzado, a pesar de ser esta la fuente máxima por excelencia para los efectos aquí planteados. Son los estudiantes los primeros receptores de la efectividad de políticas educativas o como afirma Bogue y Saunders (1992), son la pieza clave en cualquier programa efectivo de evaluación de la calidad educativa. Si este tipo de estudios se convirtiera en un proceso permanente se contaría siempre con datos actuales y específicos suficientes para la toma de decisiones razonada en relación con el cambio estructural y con la aplicación de recursos humanos y materiales. Agregaríamos a manera de especificación los recursos académicos disponibles incluyendo desde nuevo las nuevas tecnologías, sin las cuales no se comprendería la calidad educativa anhelada.

Un estudio de la Organización para la Cooperación y el Desarrollo Económicos (OCDE) indica que como en muchos paises miembros, en México un titulo de educación superior eleva las posibilidades laborales y se tiene mejor trabajo e ingresos, aunque las condiciones de trabajo han empeorado en los últimos diez años; los más jóvenes enfrentan dos serios problemas relacionados con sus competencias como la informalidad y la sobre-cualificación (OCDE, 2019)

En forma recurrente se menciona el término cambio universitario cuando se habla de calidad educativa. En este sentido se entienden las modificaciones intencionales en las reglas del comportamiento institucional, ya sean disposiciones normativas, creencias, valores o prácticas que estructuran cierto orden social universitario que iniciden directamente en la calidad universitaria. Rodolfo Tuirán (Tuirán, 2010) indica que la educación superior en el mundo es un ámbito de profundas transformaciones con reprecusiones en los planos político, social, económico y cultural.

La calidad educativa ha sido objeto clave gubernamental si bien no se han implantado mecanismos adecuados para garantizar la calidad de la educación superior que resulta compleja, fragmentada y no pocas veces escasa de transparencia.

Según el estudio antes mencionado de la OCDE, la evaluación y la acreditación de programas es voluntaria para todas las instituciones, lo que se traduce en que los programas educativos se pueden impartir aunque no haya sido sometidos a evaluación alguna. En el caso de la educación superior es competencia de los Comités Interinstitucionales para la Evaluación de la Educación Superior (CIEES) y el Consejo para la Acreditación de la Educación Supoerior (COPAES), concretamente, cuya efectividad abordaremos más adelante.

No se puede soslayar que la educación a nivel mundial ha cambiado drásticamente. Mayor matrícula, más disciplinas, acelerado proceso de generación del conocimiento y modificaciones de la estructura de la organización universitaria asociadas a la aparición de nuevas funciones de administración y gestión (Badillo, Buendía, \& Krucken, 2015). La denominada tercera misión de la universidad es buscar contribuir al fortalecimiento de la vinculación de la educación con la sociedad, a través de la participación en la solución de problemas que afectan al desarrollo económico, político, social, cultural y tecnológico.

Es así que la producción de conocimiento avanza a pasos agigantados a nuevas fases. Tradicionalmente, la comunicación entre ciencia y la sociedad fue unilateral, los científicos poseían el conocimiento experto en tanto que a los legos en la materia habia que ilustrarlos y educarlos (Gibbons, 1997). Esto ha cambiado drásticamente por el aumento del nivel educativo de la población y el uso de las aplicaciones tecnológicas, entre otros aspectos relevantes. 
En educación superior, según datos del Instituto Nacional de Estadística, Geografía e Informática (INEGI, 2003), para el ciclo escolar 2001-2002 el área de Ciencias Sociales y Administrativas registró la mayor cantidad de alumnos inscritos, egresados y titulados con porcentajes de $49 \%$, $54.7 \%$ y $53.3 \%$ respecto al total nacional.

Un estudio de Buendía, Sampedro y Acosta (Buendía \& Sampedro, 2013) aborda el tema sobre evaluación y acreditación de programas de licenciatura en México, con la pregunta expresa si con ambas se mejora la calidad de aquellas, a partir de las percepciones de los estudiantes de seis universidades mexicanas. En opinión de los alumnos, existen diversas limitaciones que no permiten sedimentar los esfuerzos que se han realizado para generar procesos de aseguramento de la calidad de los programas educativos, y los diversos elementos que los constituyen académica y admistrativamente. Los resultados refieren además que los procesos de evaluación y /o acreditación son escasamente conocidos por los estudiantes toda vez que menos de la mitad basó su elección de carrera en los sistemas de acreditación. Además, este sector tiene una opinión desfavorable acerca del uso que se hace de los resultados de las diversas evaluaciones institucionales y de la información que sure de la planeación institucional. El estudio infiere ademas que las fuentes de información con las que cuentan la mayoría de los alumnos para evaluar la relación entre la oferta laboral y y carrera, no proviene de los procesos de evaluación y acreditación. En suma, se advierte que si bien los esfuerzos institucionales son loables, lo cierto es que se requiere de un trabajo más amplio que sea incluyente con los estudiantes como centro y eje de todo proceso académico de calidad.

\section{Importancia de la percepción estudiantil sobre calidad educativa}

Los estudiantes son portadores en sí mismos de valiosa información que deseablemente debe ser aprovechada por las instituciones educativas (Rojas \& González, 2016). Se agrega que si toda institución educativa revisara constantemente su desempeño académico a partir de las percepciones de los estudiantes, se estaría en mejor posición para a)Revisar y en su caso cambiar los objetivos que plantea que logren los alumnos en cada etapa de formación b) Valorar la satisfacción de las expectativas y necesidades de formación de los alumnos c)lmpulsar metodologías de enseñanza eficaces en la promoción de la construcción del conocimiento d) Determinar la viabilidad de modificación o sustitución de contenidos y/o metodologías ineficaces o que promuevan aprendizajes de competencias no pertinentes para el ejercicio profesional.

Cabe precisar que la Universidad de Sonora cada dos años realiza una encuesta de apreciación estudiantil y la información que se capta se traduce en estadísticas que integran los indicadores institucionales prioritarios (UNISON, 2015). En suma, con la aplicación del instrumento se busca conocer el sentir de la población estudiantil acerca de la formación que se le brinda. Un primer apartado es relativo a la opinión que se tiene respecto a la planta docente; la segunda sobre aspectos de organización académica y administrativa y sobre la calidad de servicios e instalaciones básicas. Se abordan aspectos relacionados con el plan de estudios de la carrera que cursan y se les solicita que expresen su grado de satisfacción con respecto a la formación profesional que están recibiendo. En este caso el estudio es institucional lo que significa que comprende las tres Unidades Regionales ubicadas en Hermosillo (Centro) Navojoa (Sur) y Caborca (Norte) y busca conocer el sentir de la población estudiantil acerca de la formación que se le brinda.

Del documento que se menciona se extraen algunos resultados que reflejan las necesidades de los estudiantes y su grado de satisfacción con los servicios educativos que ofrece la Universidad de Sonora, con la observación de que es sobre un cuestionario ciertamente general pero que puede ser considerado como un termómetro de la percepción del alumnado.

Para muestra, se reflejan en el siguiente cuadro algunos de los resultados obtenidos respecto a la evaluación de la licenciatura en Derecho en campus Caborca sobre una población de reingreso para 2015 de 355 estudiantes; la muestra fue de 103 y el total de encuestas realizadas fueron 67: El promedio es en escala de 1 a 7 , donde 7 es la calificación más alta.

Algunos resultados de encuesta de apreciación estudiantil 2015

Cuadro 1. Elaboración propia con datos de (UNISON, 2015)

\begin{tabular}{lc} 
Rubro & Promedio \\
\hline $\begin{array}{l}\text { Indice de satisfacción de estudiantes } \\
\text { Evaluación docente }\end{array}$ & 4.9 \\
\hline $\begin{array}{l}\text { Claridad del profesor al exponer ideas y conceptos } \\
\text { Uso en clase de tecnologías de la información por los } \\
\text { docentes }\end{array}$ & 5.1 \\
\hline $\begin{array}{l}\text { Opinión estudiantil sobre motivación del docente para } \\
\text { acceder a nuevos conocimientos }\end{array}$ & 4.3 \\
$\begin{array}{l}\text { Opinión de estudiantes sobre la forma en que los docentes } \\
\text { vinculan conocimientos teóricos con la práctica }\end{array}$ & 4.9 \\
\hline $\begin{array}{l}\text { Opinión estudiantil sobre actualización de conocimientos } \\
\text { de los docentes }\end{array}$ & 4.9 \\
$\begin{array}{l}\text { Opinión de los estudiantes sobre atención de coordinador } \\
\text { de programa }\end{array}$ & 3.8 \\
\hline $\begin{array}{l}\text { Equipamiento de aulas } \\
\text { Condiciones de biblioteca }\end{array}$ & 3.7 \\
\hline \begin{tabular}{l} 
Acceso a servicios de cómputo \\
\hline
\end{tabular} & 5.6 \\
\hline
\end{tabular}


Como se observa en este primer cuadro de resultados, los promedios son ciertamente favorables a la luz del análisis realizado a nivel central por la institución. La puntuación más alta fue para reconocer las condiciones de biblioteca en tanto que la más baja para reprobar el desempeño del coordinador de programa de la licenciatura en Derecho. La evaluación docente se registró en 5.3 y la calidad del profesor para exponer ideas y conceptos fue de 5.1. Considerando ambos resultados, se observa que son buenas evaluaciones. Sin embargo, la encuesta local aplicada en los meses de febrero y marzo de 2017, que se dirigió a estudiantes de la licenciatura en Derecho del Campus Caborca refleja resultados distintos. Se consideró al total de reingreso al octavo semestre, con una población de 75, aplicándose 70 en forma efectiva.

El instrumento constó de 3 apartados: uno relativo al programa educativo y aprendizaje, otro concerniente a infraestructura académica y el tercero es una autovaloración del alumno. La escala de valoración comprendió cinco tipos de respuesta: 1. Pésimo, 2. Malo, 3. Regular, 4. Bueno 5. Excelente. Los resultados globales fueron los siguientes:

Resultados globales de encuesta aplicada a estudiantes de octavo semestre de licenciatura en derecho campus Caborca

En el desglose de resultados se advierte que el área evaluada más baja es la parte medular de la encuesta concerniente a la primera parte, Programa Educativo y Aprendizaje, en la que se abordaron aspectos relativos al programa que se les está ofertando, teniendo el menor promedio la pregunta cinco la cual reza sobre si la institución toma medidas para solucionar quejas e inquietudes que son transmitidas por los estudiantes, la cual obtuvo un 2.86 de promedio, es decir, entre malo y regular. La de mayor puntaje en este mismo apartado fue el reactivo 15: Las prácticas profesionales ayudaron a mi formación profesional, con un 4.06, es decir, los alumnos lo consideran como algo bueno.

Referente a la segunda sección denominada Infraestructura Académica, la puntuación más baja fue para la número 22: La biblioteca del campus cuenta con acervo actualizado y suficiente, con 3.36, esto es, entre regular y bueno, en tanto la má alta fue la 23: El servicio que resta el Centro de Cómputo es adecuado, con un 4.6.

Por último, se mencionan los resultados del apartado final, Autovaloración del estudiante, apartado que no aborda la encuesta institucional, y que en la local aplicada resalta la pregunta $34 \mathrm{Me}$ siento preparado académicamente respecto al nuevo sistema de justicia penal con apenas un 2.80, entre malo y regular y la de mayor valor en este sector fue la numero 33, Considero que soy un estudiante que asiste regularmente a sus clases, con un 4.46. Es decir, se califican entre bueno y excelente.
Cuadro 2. Elaboración propia con resultados de encuesta aplicada.

\begin{tabular}{|c|c|c|}
\hline Rubro & Contenido & Promedio \\
\hline $\begin{array}{l}\text { Programa } \\
\text { educativo y } \\
\text { aprendizaje }\end{array}$ & $\begin{array}{l}\text { Estructura del plan de estudio, } \\
\text { programas de materias con } \\
\text { contenido actualizado y } \\
\text { pertinente, profesores accesibles, } \\
\text { atención adecuada de las } \\
\text { autoridades, docentes con alta } \\
\text { capacidad en su desempeño, } \\
\text { suficientes herramientas } \\
\text { metodológicas recibidas, adecuada } \\
\text { orientación sobre servicio } \\
\text { social y prácticas profesionales, } \\
\text { expectativas académicas y } \\
\text { profesionales cubiertas, se recibió } \\
\text { información sobre opciones de } \\
\text { titulación }\end{array}$ & 3.46 \\
\hline $\begin{array}{l}\text { Infraestructura } \\
\text { Académica }\end{array}$ & $\begin{array}{l}\text { Aulas equipadas, instalaciones } \\
\text { limpias, biblioteca con acervo } \\
\text { actualizado y suficiente, adecuado } \\
\text { servicio del centro de cómputo y } \\
\text { centro de idiomas, satisfacción con } \\
\text { servicios administrativos, internet } \\
\text { adecuado }\end{array}$ & 3.76 \\
\hline $\begin{array}{l}\text { Autovaloración } \\
\text { del estudiante }\end{array}$ & $\begin{array}{l}\text { Dedicó el estudiante suficiente } \\
\text { tiempo a la preparación de } \\
\text { tareas, trabajos y exámenes, } \\
\text { utilizó bibliografía recomendada, } \\
\text { dedicación y responsabilidad } \\
\text { en las prácticas profesionales } \\
\text { y servicio social, administró su } \\
\text { tiempo adecuadamente entre } \\
\text { estudio y esparcimiento, dedicó } \\
\text { tiempo a la investigación y al } \\
\text { estudio adicional de temas vistos } \\
\text { en clase, se siente preparado } \\
\text { académicamente respecto } \\
\text { al Sistema de Justicia Penal, } \\
\text { recomendaría estudiar este } \\
\text { programa educativo en el campus } \\
\text { Caborca }\end{array}$ & 3.74 \\
\hline
\end{tabular}

Como se podrá advertir, si bien la escala institucional es de 1 a 7 y la local es de 1 a 5 , es evidente el grado de insatisfacción de los estudiantes del campus Caborca tanto en el Programa educativo y aprendizaje, como en Infraestructura Académica y en la Autovaloración del estudiante, aunque en este último apartado ellos consideran que son estudiantes que asisten regularmente a sus clases y que cumplen con sus tareas y obligaciones. Cabe señalar que de los setenta encuestados, 54 (77.14\%) son alumnos regulares, es decir, cursan el semestre que les corresponde sin haber reprobado materias, en tanto $16(22.86 \%)$ son irregulares. Distribuidos por género, 47 (67.14\%) son femenino y $23(32.86 \%)$ masculino.

Al final de la encuesta se designó un apartado para comentarios generales que los estudiantes pudieran realizar libremente. Destaca la queja de no contar con un plan 
de estudios actualizado, que los profesores no asisten regularmente a sus clases lo que incide en un marcado ausentismo y desmotivación estudiantil. Asimismo refieren que los espacios-aula no cuentan con mantenimiento que les permita realizar adecudamente sus labores (falta de limpieza, cupo saturado por aula, inutilidad de cañón y computadora). Existe una marcada percepción de que el programa educativo se encuentra abandonado por autoridades y maestros, aunque aclaran que no todos de éstos últimos, lo que hace que el programa educativo se sostenga.

Retomando el punto sobre acreditación, cabe precisar que el programa educativo de Derecho se encuentra evaluado favorablemente por CIEES y por COPAES a través del Consejo Nacional para la Acreditación Superior en Derecho, A.C. (CONFEDE). Si bien la evaluación externa y la acreditación de la educación han logrado superar inercias (Rubio, 2007)es evidente que se requiere analizar los resultados particulares que nos ocupan dado que se advierte que la realidad en aula, es decir, la percepción de los estudiantes difiere con los resultados institucionales tanto generales como locales.

\section{CONCLUSIONES}

Las autoridades y profesores del Campus Caborca deben analizar los resultados de las encuestas tanto institucionales como locales a la luz de la calidad deseada para el programa educativo de Derecho puesto que la percepción de los estudiantes es opuesta a los resultados institucionales y locales del Campus Caborca. Si los sistemas de evaluación y acreditación son procesos que coadyuvan de manera efectiva al mejoramiento de los servicios educativos, resulta incongruente que el programa educativo de Derecho obtenga los resultados que se presentan con esta investigación, máxime que el programa se encuentra evaluado favorablemente por CIEES y CONFEDE.

Resulta pertinente que se amplíe el nivel de habilitación de la planta académica por medio de la formación de estudios de posgrado de alta calidad, así como de la formación didáctica pedagógica acorde al nuevo modelo curricular, con orientación a la formación profesional integral, sin demérito de la atención permanente y constante en aula, dado que una de las mayores quejas de los estudiantes es el ausentismo del profesorado.

Es necesario instrumentar un plan emergente $y$ posteriormente permanente de mejora del desempeño escolar de los estudiantes universitarios, el cual se pueda medir periódicamente a fin de fortalecer el programa educativo mediante procesos efectivos internos y externos de evaluación y su uso en la mejora de aquellos para la formación profesional del estudiante. Asimismo, es insoslayable la renovación del plan de estudios de la licenciatura en Derecho a fin de que los egresados sean capaces de desempeñarse con éxito en un mercado laboral cada vez más exigente de mayes competencias profesionales

\section{REFERENCIAS}

Acosta, A. (2010). Príncipes, burócratas y gerentes. El gobierno de las universidades públicas en México. México: ANUIES.

Badillo, R., Buendía, A., \& Krucken, G. (2015). Liderazgo de los rectores frente a la "tercera misión" de la universidad. Revista Mexicana de Investigación Educativa, 393-417.

Bogue, E., \& Saunders, R. L. (1992). The Evidence for Quality. San Francisco, California, EEUU: Jossey Bass Inc. Publishers.

Buendía, A., \& Sampedro,J. (2013, diciembre). ¿̇a evaluación y acreditación mejoran la calidad de programas de licenciatura en México? Revista Reencuentro(68), 58-68.

Cruz, Y., \& Cruz, A. (2008). La educación superior en México tendencias y desafíos. Avaliacao: Revista de Avaliacao da Educacao Superior (Campinas), 23-29.

Gibbons, M. e. (1997). La nueva producción del conocimiento. Barcelona: Pomares-Corredor S.A.

INEGI. (2003). Instituto Nacional de Estadística, Geografía e Informática. Retrieved from http://internet. contenidos.inegi.org.mx/contenidos/Productos/ prod_serv/contenidos/espanol/bvinegi/productos/ historicos/2104/702825460679/702825460679_1.pdf

Muñoz, H. (2016). ¿Hacia dónde va la Universidad dē Siglo XXI? México: MAPorrúa.

OCDE. (2019). OCDE.ORG. Retrieved from OCDE.ORG:

https://www.oecd.org/centrodemexico/medios/educacion_ superior_en_mexico.pdf

Rubio, J. (2007, diciembre). La evaluación y acreditación de la educación superior en México: un largo camino aún por recorrer. Reencuentro, 50, 35-44.

SEGOB. (2020, febrero 11). Diario Oficial de la Federación. Retrieved from SEGOB-DOF: http://dof.gob.mx/nota detalle.php? codigo $=4642789 \&$ fecha $=29 / 01 / 1990$

Tuirán, R. (2010). Aliat.edu. Retrieved from Aliat.edu: http://online.aliat.edu.mx/adistancia/Calidad/unidad4/ lecturas/TXT_1_S4_EDUC_SUP_AVAN_REZ_RET_ TUIRAN.pdf

UNISON. (2015). Universidad de Sonora. Retrieved from Planeación.unison.mx: https://planeacion.unison.mx/ pdf/encuesta_de_apreciacion_estudiantil_2015.pdf

Yzaguirre, L. (2005). Calidad Educativa e ISO 9001-2000 en México. Revista Electrónica Iberoamericana sobre Calidad, Eficacia y Cambio en Educación, 3(1). 